\title{
Localized primary sclerosing cholangitis mimicking a cholecystectomy stricture relieved by an endoprosthesis
}

\author{
Ran Oren ${ }^{1}$, Eran Goldin², Noam Harats' ${ }^{1}$ Eugene Libson ${ }^{3}$ and Daniel \\ Shouval ${ }^{1}$
}

\author{
${ }^{1}$ Liver Unit, Department of Medicine A, Departments of ${ }^{2}$ Gastroenterology and ${ }^{3}$ Radiology, Hadassah \\ University Hospital, POB 12000, Jerusalem 91120, Israel.
}

\begin{abstract}
Summary: Primary sclerosing cholangitis presenting as a localized stricture affecting a segment of the extrahepatic biliary tree is rarely found. We describe the case of a 39 year old woman with obstructive jaundice, in whom this diagnosis was proven by endoscopic retrograde cholangiography. An endoprosthesis was endoscopically introduced through the stenotic area which led to a dramatic improvement enabling a successful liver transplantation after 2 years.
\end{abstract}

\section{Introduction}

Primary sclerosing cholangitis (PSC) is a relatively rare disease of unknown aetiology. ${ }^{1-6}$ Following the original description by Delbet in 1924, fewer than 80 cases were reported until $1970 .^{3}$ The improvement in cholangiographic techniques such as endoscopic retrograde cholangiography (ERCP) has increased the rate of detectable disease $^{7}$ without resorting to laparotomy. ${ }^{3,8-11}$ The characteristic obliterative, inflammatory fibrosis usually involves the whole biliary tree, and less commonly, the changes may be localized to a segment of the extra-hepatic bile ducts. ${ }^{1-3}$

Stenosis of the bifurcation of the common hepatic duct is usually caused by cholangiocarcinoma arising in the wall of the duct or by a neoplastic invasion of the hilar lymph nodes. ${ }^{12}$ Nevertheless, stenosis can also be caused by sclerosing cholangitis. We report a case of PSC mainly localized at the bifurcation of the common hepatic duct and emphasize the therapeutic advantage of biliary endoprosthesis insertion in these cases.

\section{Case report}

A 39 year old female patient was admitted for evaluation of 'chronic liver disease' which had started 3 years before. Her first symptoms included severe pruritus followed by jaundice. There was a mild elevation of bilirubin $41 \mu \mathrm{mol} / 1$ (normal

Correspondence: Ran Oren, M.D.

Accepted: 2 August 1990
$2.5-17 \mu \mathrm{mol} / \mathrm{l})$ and alanine amino transferase (ALT) $135 \mathrm{U} / 1$ (normal < $35 \mathrm{U} / \mathrm{l}$ ) accompanied by a marked elevation of alkaline phosphatase. On ultrasound examination no evidence for dilated extrahepatic bile ducts was seen. Explorative laparotomy, 3 years prior to her admission, revealed a moderately enlarged liver, an inflamed gallbladder with no evidence of gallstones. Cholecystectomy was performed despite the absence of gallstones. An open liver biopsy revealed severe extrahepatic cholestasis. The postoperative course was uneventful, while bilirubin and ALT values returned to normal level. The alkaline phosphatase remained, however, markedly elevated.

Five months prior to her current admission, severe cholestasis and jaundice reappeared. A needle liver biopsy at that stage again revealed evidence for extrahepatic cholestasis. A trial of prednisone treatment did not cause any significant improvement. At that stage the patient was transferred to our hospital.

Upon her admission she appeared deeply jaundiced with a Cushingoid face, her temperature was $38.5^{\circ} \mathrm{C}$. A tender liver was palpated $5 \mathrm{~cm}$ below the costal margin. The erythrocyte sedimentation rate was $110 \mathrm{~mm}$ (first hour, Westergen). The white blood count was $11.5 \times 10^{9} / 1$ with a shift to the left. The haemoglobin was $9.7 \mathrm{~g} / \mathrm{dl}$ with normocytic normochromic indexes. Bilirubin was $143 \mu \mathrm{mol} / 1$ (normal $=2.5-17$ ), transaminase $1505 \mathrm{U} / 1$ (normal $=7-35$ ), alkaline phosphatase 1865 units (normal $=30-85$ ), and gamma glutamyl transpeptidase up to $2650 \mathrm{U} / 1$ (normal $=10-40$ ). Albumin was $23 \mathrm{~g} / \mathrm{l}$ with globulin $47 \mathrm{~g} / \mathrm{l}$.

Abdominal ultrasound and computerized axial tomography were within normal limits. A revision 
of the intraoperative cholangiogram from the operation done 3 years ago showed an irregularity in the distal right hepatic duct, while the left hepatic ducts were not demonstrated at all. An ERCP was performed in which the common bile duct appeared normal at its lower two thirds. In the upper third, there was a complete obstruction of the common hepatic duct, in the bifurcation area, that did not permit visualization of both intrahepatic bile ducts (Figure 1). Therefore, papillotomy was performed and an endoscopic endoprosthesis (14 cm length, 10F diameter) was introduced into the right hepatic duct through the obstruction. Injection of contrast material through the stent revealed a normal right hepatic biliary system, but the left one was not visualized (Figure 2). The stent was left in order to decompress the congested biliary tree. Following insertion of the endoprosthesis, jaundice, pruritus, abdominal tenderness and the disturbed liver function tests improved dramatically. Except for an early episode of Gramnegative sepsis that was controlled by antibiotics, no further complications were recorded.

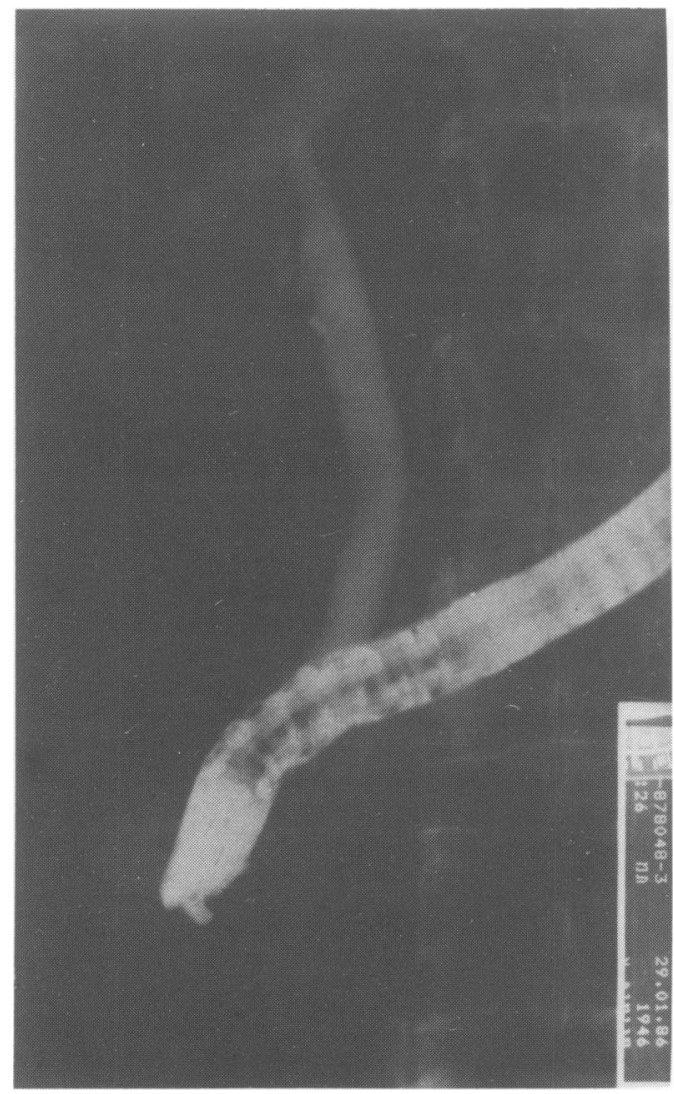

Figure 1 Endoscopic retrograde cholangiography: a complete obstruction of the bifurcation area.

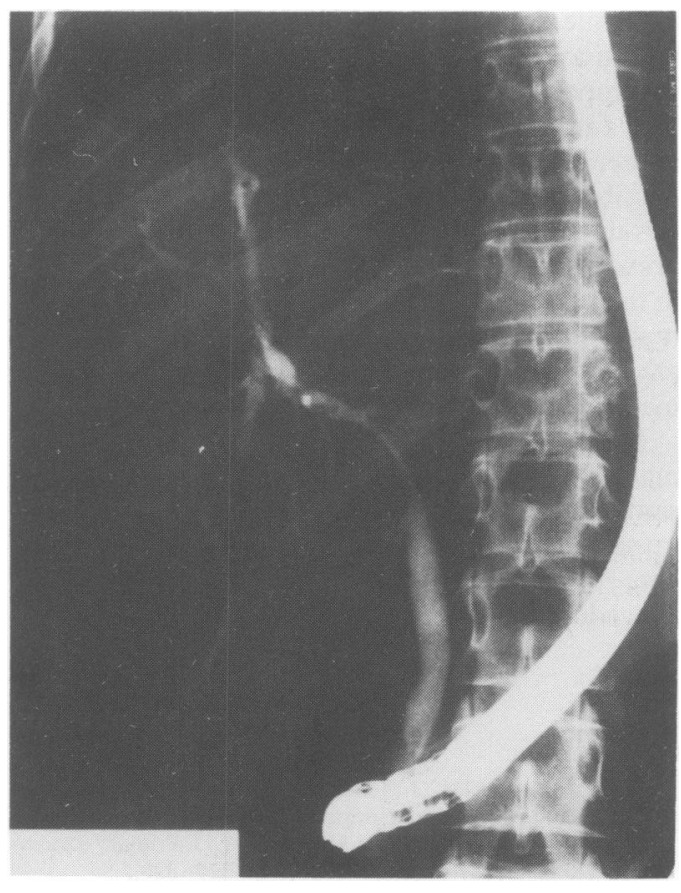

Figure 2 Endoscopic retrograde cholangiography, injection into the right hepatic duct. Normal intrahepatic ends. Structure in the bifurcation area.

Nine months following the procedure the patient was stable without fever, jaundice or pruritus, but the alkaline phosphatase was still elevated. After 24 months the patient underwent a liver transplantation.

The native liver should sclerosing cholangitis complicated by early cirrhosis, superinfection giving microabscesses. There was no evidence of tumour.

\section{Discussion}

Our patient represents an example of the rare variant of localized PSC with demonstration of a promising palliative treatment. The differential diagnosis of a localized biliary stenosis in our patient included choledocholithiasis, cholangiocarcinoma (Klatchkin tumour) or a post-operative stricture. $^{12}$ The radiological picture ruled out the possibility of a stone. Alternatively, post-operative stenosis was ruled out due to the obstructive feature of the disease prior to surgery, as also confirmed by the intra-operative cholangiogram. Therefore, the bile duct stenosis in our patient was interpreted to be the result of localized PSC.

Although the ERCP results were compatible with a Klatchkin tumour, and the development of 
high bile duct stricture in patients suffering from PSC may indicate that a bile duct carcinoma has developed, the long, relatively benign clinical history and the negative CT scan were all against the existence of a malignant tumour. Nevertheless, the possibility of a tumour cannot be ruled out with certainty, because such tumours are extremely slow growing and do not show up on the CT scan, except in the late stage. From the point of view of biopsy, it should also be kept in mind that these tumours are very fibrous and the biopsy may fail to reveal a malignant picture even if it exists.

In the present case, diagnosis and treatment were made by ERCP, a procedure that allows, at the same time, diagnosis and the palliative treatment of introducing a biliary endoprosthesis. In fact, in the case of localized PSC at the bifurcation, the procedure of choice would be the introduction of

\section{References}

1. Panes, J., Bordas, J.M., Bruguera, M. et al. Localized sclerosing cholangitis. Endoscopy 1985, 17: 121-122.

2. Utley, R.J., Angus, H.B. \& Macbeth, W.G. Primary sclerosing cholangitis. NZ Med J 1984, 310: 324-326.

3. Chapman, R.W. Primary sclerosing chlolangitis. J Hepatol 1985, 1: 179-186.

4. Larusso, N.L. Primary sclerosing cholangitis. $N$ Engl J Med 1984, 14: 899-903.

5. Summerfield, J.A. Primary sclerosing cholangitis. Postgrad Med J 1983, 59: 99-105.

6. Smadja, G., Bowley, N.B., Benjamin, I.S. et al. Idiopathic localized bile duct strictures: relationship to primary sclerosing cholangitis. Am J Surg 1983, 148: 404-408.

7. Cameron, J.L., Gayler, R.W., Herlong, H.G. et al. Sclerosing cholangitis: biliary reconstruction with silastic transhepatic stents. Surgery 1983, 93: 324-330.

8. MacCarty, R.L., LaRusso, N.F., Wiesner, R.H. et al. Primary sclerosing cholangitis: findings on cholangiography and pancreatography. Radiology 1983, 149: 1298-1301. two endoprostheses, one to each liver lobe. Such dual decompression should lead to bilateral liver lobe drainage and avoid sepsis and cholangitis of the non-drained side. In our case, repeated attempts to introduce a second stent into the left side failed due to the severe stenosis. The efficiency of the internal stent for decompression of the biliary system is well documented, ${ }^{9-11}$ but its experience in localized PSC is limited due to rareness of the diseases. ${ }^{1,9-11,13} \mathrm{~A}$ better awareness for the possibility of a localized variant of PSC (despite a past history of cholecystectomy) and the increasing utilization of ERCP in cholestatic jaundice may help in diagnosis and palliation in those patients, even if the relief is only temporary. This procedure provides a better chance for a future liver transplantation. However, the ERCP should have been performed sooner in the case presented.

9. Curzio, M., Bernaschan, G., Gullotta, R. et al. Association of ulcerative colitis, sclerosing cholangitis and cholangiocarcinoma in a patient with IgA deficiency. Endoscopy 1985, 17: 123-125.

10. Carole, B.A. \& Oppenheimer, O.A. Sclerosing cholangitis: sonographic demonstration of bile duct wall thickening. $\mathrm{Am}$ J Roentgenol 1982, 139: 1016-1018.

11. Wiesner, R.H. \& Larusso, N.F. Clinicopathologic features of the syndrome of primary sclerosing cholangitis. Gastroenterology 1980, 79: 200-206.

12. Golematis, B., Giannopoulos, A., Papachriston, D.N. et al. Sclerosing cholangitis of the bifurcation of the common hepatic duct. Mt Sinai J Med (NY) 1982, 49: 38-45.

13. Johnson, G.K. Endoscopic treatment of biliary duct strictures in sclerosing cholangitis; follow up assessment of new therapeutic approach. Gastrointest Endosc 1987, 33: 1-12. 